\title{
The quantum computer in the subconscious - A new dimension
}

\section{Opinion}

Our brain has two major subdivisions: Conscious located on the surface of the brain and Subconscious located in deeper areas of the brain. The name Subconscious besides reflecting the fact that the Subconscious is located under the conscious, it reflects also the fact that we don't have conscious awareness of its work. Since the Emotional brain, called Limbic System, is in the deeper areas of the brain, the Subconscious rules our emotions.

Our organs are also subordinated to the Subconscious and for this reason we don't have conscious awareness of their functioning. It is deliberately done so because if our life is threatened and we need to make important decisions to survive, we don't want to be bothered with information about the functioning of our organs. However, we are going to show that many branches of alternative medicine operate on the basis of the connection organs - Subconscious.

Hypnosis and head trauma revealed ${ }^{1}$ that the Subconscious has: 1) tremendous storage capacity (it has a full record of everything we have seen, heard, smell, and emotionally experienced during our lifetime) and 2) a very high speed of computation. ${ }^{1}$

In a previous article, ${ }^{2}$ the author explained that a detailed lifetime record in the Subconscious requires a compact record and such is the holographic record. Also, the fact that all organs, which are subordinated to the Subconscious, are holographically represented on the palms, the feet, the ears, and the eyes ${ }^{3}$ illustrates that the Subconscious operates on the holographic principle.

Russian studies ${ }^{4}$ showed that seeing with the mind is done through the Subconscious and is also holographic. The person was seeing the object when blindfolded and when signal was not recorded from the eye to the brain. He was seeing the object as three dimensional, which means holographic vision. Since holographic images are possible only with coherent laser type of waves, this means that our Subconscious operates on wave basis, while our conscious operates on material basis.

Farther Russian studies ${ }^{4}$ proved that when seeing with the mind the light perception of the mind has torsion nature. Torsion generator was created, which didn't emit any light or electromagnetic waves. However, person seeing with his mind could see if the generator is active or turned off and could draw the diagram of the direction of field emission. These torsion waves carry information and when they are slowed down to the speed of light, this is the light of electromagnetic nature, which we see with our eyes.

The author illustrated in previous article ${ }^{5}$ that one of the ways acupuncture works is through the waves generated at acupuncture treatments. The pulse diagnosis, ${ }^{5}$ the iris diagnosis ${ }^{6}$ and the voice diagnosis, ${ }^{7}$ used in alternative medicine, are also based on waves, ${ }^{8}$ which are parts of our dynamic nonlinear electromagnetic field (NEMF). Since our Subconscious operates on wave basis, it seems that our NEMF rules the functioning of the whole body from the Subconscious.

To the subconscious are also related the psychic abilities, which some people have. These psychic people can predict events in the future or see the past, which are time-leaps to the future and the past.
Volume II Issue 4 - 2018

\author{
Maria Kuman, PhD \\ Holistic Research Institute, USA
}

Correspondence: Maria Kuman, Holistic Research Institute, I 4 I 4 Barcelona Dr, Knoxville, TN 37923, USA, Email holisticare@mariakuman.com

Received: June 29, 2018 | Published: July 10, 2018

There is no reason (cause) why this psychic person should be able to see the future and the past. If so, the Subconscious does not obey the causality principle.

Thus, the Subconscious has an enormously high rate of computation and does not obey the causality principle. If computations are done in the Subconscious with enormously high speed, this is because the torsion waves of the Subconscious that carry information can propagate with a speed higher than the speed of light. If so, probably the relativistic theory of Einstein should be expanded the high-speed processes in the Subconscious.

The relativistic theory of Einstein is a dynamic theory and dynamic is best described if time is an active variable. Based on this, Minkovski added a time variable to the three-dimensional space representation. This four dimensional representation allowed him to re-write all Einstein formulae in very symmetric and simple forms.

In Minkovski representation, our world is divided into two sets of two cones (Figure 1). One set of cones is called "The Real Word", in which nothing can move with a speed higher than the speed of light $\mathrm{c}$ in vacuum. The time is one-dimensional, runs from past to future, and nothing can happen without a cause. They are called "light cones" and in them all events obey the causality principle.

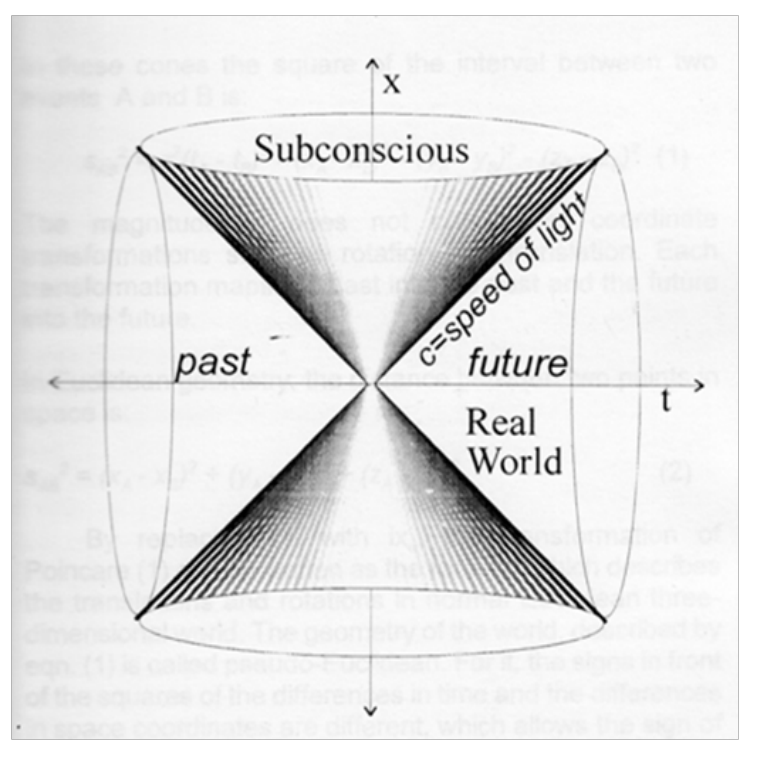

Figure I Minkovski's Four- Dimensional Representation of Einstiens Relativistic Theory. 
The other set of two shaded cones represent a strange word, called "Elsewhere", in which processes are allowed with a speed higher than the speed of light. Also, in this world called "Elsewhere", the events do not obey the causality principle, which means things can happen without a cause. It allows time leaps to the past and to the future.

Nobody wanted to deal with this messed up world and these cones remain unused. However, the bizarre properties of the Subconscious seem to be a perfect match to the bizarre properties of this messed up world called "Elsewhere". The Subconscious, just like the "Elsewhere" does not obey the causality principle, allows time leaps to the past or future, i.e. allows seeing the past and predicting the future, and allows speeds higher than the speed of light.

So, the idea is to use the second (shaded) pair of cones in the Minkovski representation to describe the quantum Subconscious. An appealing feature of the Minkovski representation is the possibility for communication between these two so dramatically different worlds - the "Real World" and the "Elsewhere", which in our case means communication between the Conscious and the Subconscious.

At the border between the two worlds there are closed curves and null curves, which allow communication between the two worlds. In our case, these curves would describe the potential possibility for communication and exchange of information between our Subconscious ("Elsewhere") and the Conscious ("The Real World").

If exchange of information between the "Elsewhere" (the Subconscious) and the "Real World" (the Conscious) is really possible, then strange for the "Real World" events like psychic events or holographic seeing with the mind would be no problem to explain. Also, the validity and value of different modalities of alternative medicine, like acupuncture, pulse diagnosis, voice diagnosis, and iris diagnosis are not questionable anymore because they relate to the waves of the human NEMF, which operates at subconscious level.
Also, now when we know the principles on which our quantum computer in the Subconscious operates, we can create quantum computers on the same basis. Einstein couldn't accept the quantum theory for a very long time. With the new development of science, the relativistic theory can now be united with the new quantum theory, which will be the basis of the new quantum computers.

\section{Acknowledgements}

None.

\section{Conflict of interest}

The author declares no conflict of interest.

\section{References}

1. Kuman M. A Guide to the Mind's Secrets. Health and Happiness Books; 1996.

2. Kuman M. Research on Medicine and Engineering Science. 2018:2(5).

3. Kuman M. Advances in Complimentary and Alternative Medicine. 2018:2(4).

4. Tihoplav V, Tihoplav T, Cosnanie Vselenoi. (The Conscious of the Universe). Russia: Krilov; 2007.

5. Kuman M. Acupuncture and Electro-therapeutic Research. 2017;42(2):97-112.

6. Kuman M. Advances in Complimentary and Alternative Medicine. 2018:2(4).

7. Kuman M. Journal of Natural and Ayurvedic Medicine. 2018:2(3).

8. Kuman M. Modern Aspects of Ancient Acupuncture. Health and Happiness Books; 1997. 